Article

\title{
digiMe: An Online Portal to Support Connectivity through E-Learning in Medical Education
}

\author{
Si Fan * (D), Jan Radford (D) and Debbie Fabian \\ Faculty of Education, University of Tasmania, Launceston, Tasmania 7250, Australia; \\ J.Radford@utas.edu.au (J.R.); debbie.fabian@gmail.com (D.F.) \\ * Correspondence: Si.Fan@utas.edu.au \\ Academic Editors: Elizabeth Cummings and Carey Mather \\ Received: 19 May 2017; Accepted: 5 September 2017; Published: 8 September 2017
}

\begin{abstract}
Connectivity is intrinsic to all aspects of our life today, be it political, economic, technological, scientific, or personal. Higher education is also transcending the previous paradigm of technology enabled content delivery and e-learning, with a new emphasis on connectivity, enabling participants to exchange knowledge and collaborate to meet educational goals. In this study, a social media technology supported website-digiMe-was developed and evaluated at the School of Medicine of one Australian university. Connectivity to other medical learners and health professionals is intrinsic to digiMe. This paper reports the functionalities of this website, results of a post-intervention evaluative survey, and statistics of website usage generated from Google Analytics. The results revealed more active adoptions and a more positive attitude towards digiMe from Year 4 students compared to Year 5 students. The participants showed a desire for access to a recommended collection of apps, such as those offered through digiMe. However, many participants did not use digiMe beyond initial introduction to it. digiMe demonstrated its potential in raising awareness of web and mobile apps useful for enhancing connectivity, although it needs to be introduced to students in earlier years of their medical education to achieve a higher impact on their learning.
\end{abstract}

Keywords: digiMe; online portal; medical education; e-learning

\section{Introduction}

Connectivity is intrinsic to all aspects of our life today, be it political, economic, technological, scientific, religious, cultural, societal, or personal. It has become prominent across workplaces and education in general. This connectivity has been acknowledged by the Educause Learning Initiative who stated that "Higher education is entering a new, evolutionary phase defined by connections between everything and everyone" [1] (p. 1). This ecosystem of technologies supports sharing and collaboration, and enables global connectivity to specialists and students across disciplines [1]. This paradigm shift can also be observed in the field of medical education though the emergence of Web 2.0 supported interactive websites [2]. The emphasis is shifting from the previous paradigm of technology enabled content delivery to integrated and personalised learning that enables all participants to create new content in individual ways to meet educational goals. In responding to this change, this study developed and trialed a Web 2.0 supported website-digiMe_allowing medical students and health professionals to make connections with other members in the learning community.

There were two stages to the larger scope study, which was conducted over a one year period. Stage 1 is the pre-intervention stage, which investigated the types of devices and Web 2.0 tools used by students and teaching staff in the School of Medicine at one Australian university, together with reasons for their adoption. The findings of this first stage can be found in another article [3]. Stage 2 is 
the post-intervention stage, in which a Web 2.0 supported website-digiMe-was developed, trialed, and evaluated. This paper reports the findings of the second stage. The findings discussed in this paper include three key sections: functionalities of the website, website evaluation obtained through a post-intervention survey, and statistics of website usage generated through Google Analytics.

\section{Literature Review}

The past decade has seen the emergence and widespread use of Web 2.0 technology and Web 2.0 supported websites, in the field of higher education in general and medical education in particular. The accessible and customisable nature of Web 2.0 tools presented opportunities for their use in medical education [4,5]. This study was shaped by two theories: connectivism [6] and the domestication theory [7], both of these are discussed below. Lastly, this section will also provide a brief review on existing Web 2.0 supported websites which were examined prior to the development of digiMe.

First, connectivity is one of the most significant values Web 2.0 technologies have brought to learning. As an alternative to existing learning theories (e.g., behaviorism, cognitivism, and constructivism), Siemens proposed connectivism as an additional learning theory, to reflect the rapidly growing knowledge and information exchange in the digital age [6]. With their collaborative, open and contributory nature, Web 2.0 technologies are a good fit for interactive, student-centred, constructivist, and negotiated pedagogies [8]. The interactive website developed in this study-digiMe-is a personalised virtual learning environment which links to and makes accessible a range of Web 2.0 tools. It recognises that pedagogical change-including greater autonomy, diversity, openness, and connectedness-is required for any personal learning environments to bring maximum benefit to education [9]. digiMe also recognises the importance of learning from other people's experiences. As stated by Stephenson [10], "since we cannot experience everything, other people's experiences, and hence other people, become the surrogate for knowledge ... 'I store my knowledge in my friends' is an axiom for collecting knowledge through collecting people (p. 1)". In particular, digiMe supports connectivity and connected learning, enabling users to "... use connections to find answers, seek out mentors and experts, investigate procedures, experiment with possibilities, and develop competencies" [1] (p. 2).

Second, this research was conducted in light of the domestication theory [7], which describes the process in which technologies are introduced into the daily practice of the potential user community, adapted and appreciated, and then shaped by the feedback of users. Developed initially in the 90s, the domestication theory has since become widely used in innovation literature, as an approach to understand the role of users in technology development and how technologies are adapted by users in their everyday practice $[7,11]$. Shaped by the domestication theory, this study developed and introduced digiMe to a target audience group, including medical students at the undergraduate level, and health professionals in the local medical learning community. A user analysis confirming the interests and needs of this group was conducted prior to the website design and implementation [3]. Both their feedback prior to and after the website development was considered as important in shaping the direction of the study and the final product of digiMe.

As in the early stages of any website development, an examination of existing tools and resources is important. At the initial stage of this study, an analysis was also undertaken to examine websites that are currently available with the aim to meet learning and information needs of the medical and health profession. Examples of these include GPaedia [12,13], e-healthspace [14], ThinkGP [2], and auspharmacist [15]. These reviewed websites provide some insights into the needs of the medical profession through their focus on membership authentication, networking, and communication with peers, and sharing of knowledge and expertise. These websites are offered to different target user groups. For instance, GPaedia and ThinkGP both included medical students and staff at the undergraduate (UG) level within their target audience, e-healthspace however only allows access for health professionals. These websites also have different focuses in terms of functionality. For instance, GPaedia's primary aim was as a repository for sharing teaching resources, Think GP provides access to 
learning programs in addition to resource sharing. Apart from e-healthspace which is cross disciplinary, the other three websites all have a more specific focus. Both GPaedia and ThinkGP have a specific subject focus on general practice; auspharmacist, on the other hand, has a specific subject focus on pharmacy. From the perspective of ongoing maintenance, e-healthspace, Think GP, and auspharmacist are bespoke systems that require considerable ongoing operational investments. GPaedia relied on volunteer curators from the learning community to maintain the website operation, however, it did not go beyond the pilot stage and was decommissioned after the project period. Lastly, all these websites are largely focused on the end products (e.g., information, knowledge), rather than the learning process. Lessons learned at this review stage were taken into consideration during the development of digiMe in this project.

\section{Methodology}

The study uses a mixed-method research approach, involving both quantitative and qualitative methods. As mentioned in earlier sections, the larger scope study included two stages, and this paper reports the methodology and results from the second stage. In this Stage 2, digiMe was developed, a post-intervention survey was used to collect both quantitative and qualitative data, and Google Analytics was also used to gather website usage data.

\section{1. digiMe Development and Presentation}

digiMe was presented as a personalisable dashboard with Web 2.0 tools that enable communication, collaboration and co-operation (see Figure 1). It provides information and links to mobile apps, however, these tools are used from the apps that users downloaded onto their devices, rather than within digiMe itself. digiMe includes screenshots, information about, and links to a total of 41 apps and social media tools. These tools were categorised into 16 functional categories including: clinical learning $(n=41)$, file sharing and storage $(n=4)$, scheduling $(n=2)$, collaborative authoring $(n=2)$, document editing and annotation $(n=8)$, audio capturing/editing/sharing $(N=4)$, slide presentations $(n=2)$, e-readers $(n=1)$, clinical practice $(n=5)$, note-taking $(n=3)$, bibliographic management $(n=2)$, audio/video conferencing $(n=1)$, social/professional networking $(n=5)$, feed readers $(n=3)$, video capturing/editing/sharing $(n=2)$, and web development and blogging $(n=1)$. digiMe also has the capacity for individualised learning. This can be achieved through: creating a favourites dashboard, a page that identifies what is new, the capacity to add bookmarks, a link to the digiMe community on Yammer (see Figure 2), and a learning space that provided categorised links to useful websites.

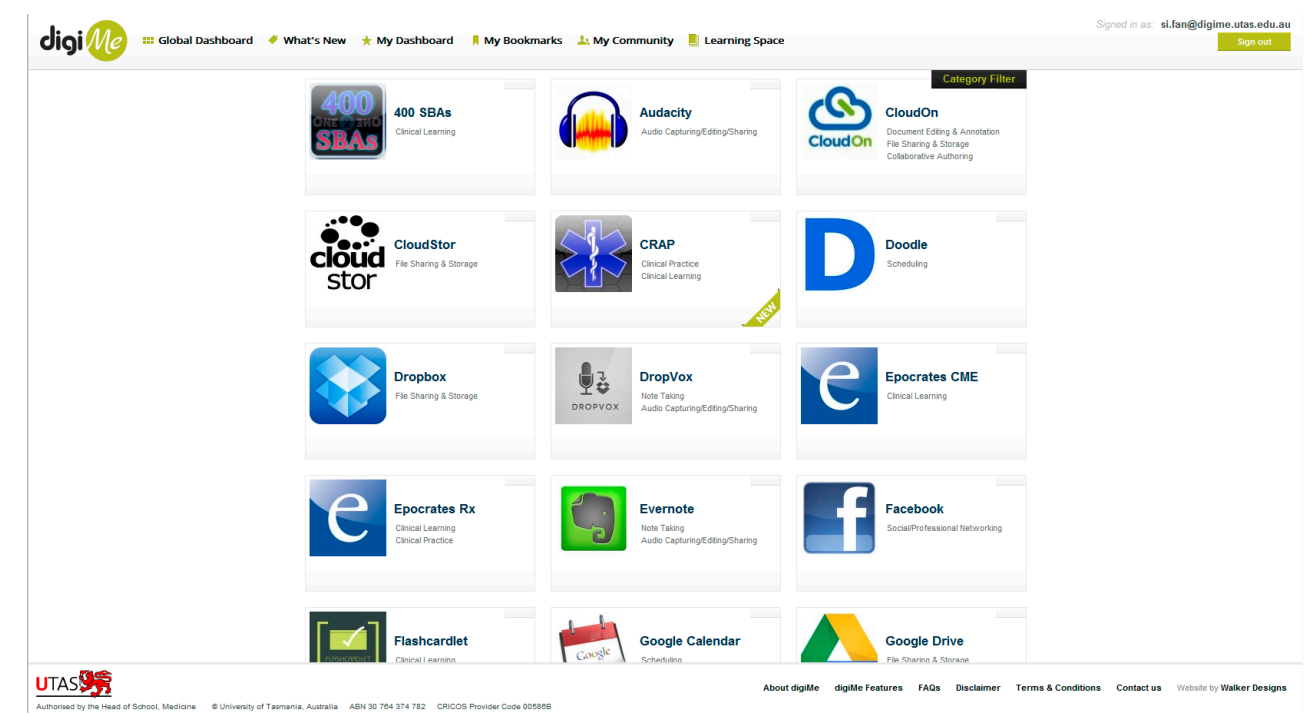

Figure 1. digiMe Global Dashboard interface. 


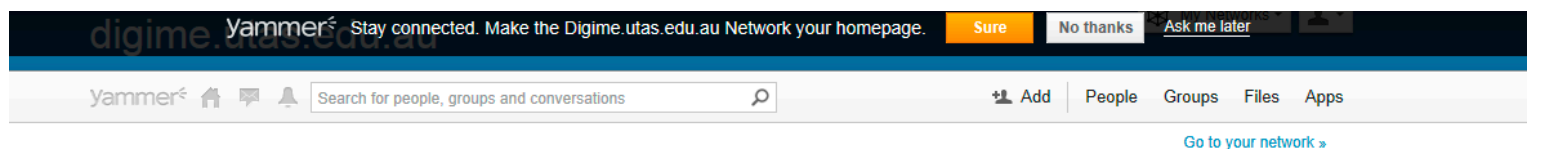

\section{Add colleagues and start collaborating}
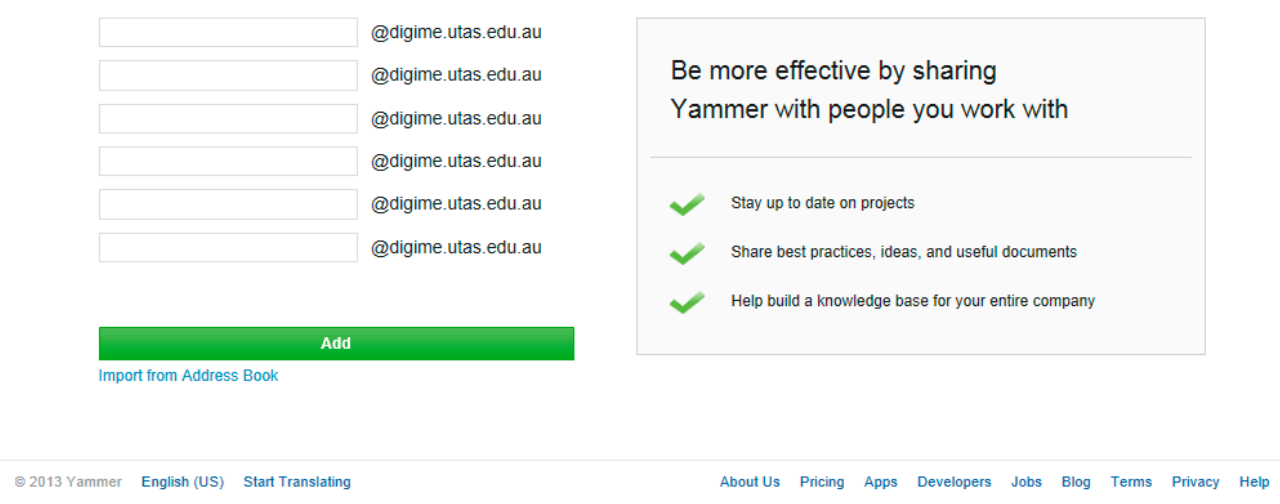

Figure 2. digiMe 'My Community' page.

\subsection{Post-Intervention Survey: Development, Validation, and Analysis}

The post-intervention survey was developed into three sections. Section A has five questions in relation to participants' demographic information. Section B included 24 questions, asking about the technologies (e.g., browsers and apps) within digiMe used by the participants. This section has a few summative-scale items, but most of the questions in this section were designed with a Likert-scale format, using the standard five-point Likert-scale: Strongly agree to Strongly disagree [16]. Section C has three open-ended questions, which asked: (1) ways in which the introduction of digiMe has contributed to the participants' learning or teaching; (2) ways in which the introduction of digiMe has improved their clinical and professional practice; and (3) other comments in relation to the introduction of digiMe.

The draft survey was tested and validated through a pilot study, which involved a group of medical students, teaching staff and experienced researchers. In particular, the content validity and the time commitment in completing the survey was examined and discussed with each of these participants. Rather than collecting their responses to the survey questions, instead, the focus was to ask for their feedback on the clarity of the question items, and the appropriateness of the length of the survey. Revisions were made in light of their suggestions for improvement.

The statistical data collected through the first two sections of the post-intervention survey were analysed using SPSS as a tool. Both Section A and Section B were analysed using descriptive analysis methods, including frequencies, and mean and median values. For the Section B items, the Kruskal-Wallis test and Mann-Whitney $U$ test were also used to examine whether statistically significant differences existed between the different participant groups [17]. For the textual data collected through Section C of the survey, a constructivist grounded theory approach was used to identify patterns in the answers to these questions [18]. Themes were generated by pulling out the frequently emerged words and phrases.

\subsection{Data Collection: Post-Intervention Survey and Google Analytics}

Access to digiMe required registration, and digiMe was only introduced to students and teaching staff at one of the clinical schools at the School of Medicine of one Australian university, and some local general practitioners and clinicians at a local hospital. There were a total of 129 registered digiMe users. All these users were invited to participate in the post-intervention survey, through 
a link to the online survey advertised on the digiMe homepage. Sixty-four of the registered users completed this post-intervention survey. The respondents consisted in the main of medical students $90.6 \%(n=58)$, with clinical teachers at $6.3 \%(n=4)$ and administrative staff at $1.6 \%(n=1)$. This role distribution reflects the population in the chosen medical education context, although clinical teachers and administrative staff presented only a small proportion in the survey sample. The analysis did not see statistically significant difference between the views of the different role groups. Therefore, the survey results in this paper considered the three role groups as a whole, rather than presenting analysis on any role group in particular. Over $89 \%$ of respondents fell in the $20-29$ age group, with $11 \%$ of them in the age group of 30 and over, this reflects the fact that the majority of them were medical students in the undergraduate degree.

In addition to the post-intervention survey, a report was generated from Google Analytics, which is a freemium analytics service launched by Google that tracks and reports website usage data [19]. The data generated included the count of visits to the website, types of devices used to access the website, and statistics on the use of apps associated with digiMe. These usage data are reported in the 'Findings' section below, along with the results from the post-intervention survey.

\section{Findings}

These findings include two major components. One of these parts presents the findings of the post-intervention survey, with the other part discussing the usage statistics recorded through Google Analytics.

\section{Findings from Post-Intervention Survey}

Section A of the post-intervention survey asks about the participants' demographic information. Within the 64 survey respondents, nearly $95 \%(n=60)$ of these participants had smartphones, predominantly iPhones $(n=40)$, and nearly $40 \%(n=25)$ additionally used computing tablets to assist them with their learning, teaching, or professional practice. Nearly half $(n=30)$ of these participants agreed or strongly agreed that they would like access to a recommended collection of apps that have been curated by suitably qualified persons. However, their interest in digiMe, which aimed to do this, was not of a commensurate level. That is, introduction to digiMe raised awareness of web and mobile apps for learning, teaching, clinical, or professional practice for only $17 \%(n=11)$ of the participants, and approximately more than half of them $(n=38)$ declared that digiMe did not raise their confidence in using similar tools. The current use of digiMe's social and professional networking tool: Yammer, was lower than expected $(11 \%, n=7)$; however, its perceived potential to be useful professionally by the medical education community was greater $(30 \%, n=19)$.

Section B of the survey examined the types of web or mobile apps the participants would like to be recommended to. The Web 2.0 tools linked to digiMe were categorised into 16 types according to their purposes. Within these 16 types, the participants held a more positive view on eight of them (see Table 1). In particular, these participants were mostly interested in being introduced to those tools which would facilitate clinical learning (mean $=4.00)$, clinical practice (mean $=4.00)$, and note taking (mean $=4.00)$. Their views on the tools for document editing or annotating $($ mean $=3.50)$, slide presentations $($ mean $=3.00)$, scheduling $($ Mean $=3.00)$, file sharing or storing $($ mean $=3.00)$ or social/professional networking (mean $=3.00$ ) were also more positive compared to the other tools listed. On the other hand, the remaining types of tools only received a relatively low interest from the participants: web development or blogging, collaborative authoring, e-Reading and feeds, bibliographic management, audio/video conferencing, audio capture/editing/sharing, and video capture/editing/sharing. This is demonstrated through the mean values of these items being lower than 3.00 . 
Table 1. Types of tools perceived more positively by the participants.

\begin{tabular}{ccc}
\hline Purpose of Web or Mobile Apps & Number of Participants Selecting Strongly Agree or Agree & Mean * \\
\hline Clinical learning & $64 \%, n=41$ & 4.00 \\
Clinical practice & $58 \%, n=37$ & 4.00 \\
Note taking & $47 \%, n=30$ & 4.00 \\
Document editing or annotating & $37 \%, n=24$ & 3.50 \\
Slide presentations & $34 \%, n=22$ & 3.00 \\
Scheduling & $34 \%, n=22$ & 3.00 \\
File sharing or storing & $33 \%, n=21$ & 3.00 \\
Social/professional networking & $30 \%, n=19$ & 3.00 \\
\hline
\end{tabular}

* Items were rated on a five-point Likert-scale: Strongly agree $=5$; Agree $=4$, Neutral $=3$, Disagree $=2$,

Strongly disagree $=1$.

Section $C$ of the survey included three open-ended questions. The answers to these questions revealed that many did not use digiMe beyond initial introduction to it. One of the reasons for this is the perceived difficulties associated with the registration process and many forgot their login details and there needed to be a clearer and easier way to reset their password. With regards to digiMe's professional/social networking, its Yammer community, a few students declared that it had nothing to offer that Facebook did not and that once they had reached their clinical years (Year 4 and Year 5), they were well established in Facebook communities, so introduction to such a tool would have to happen in their earliest student years to be potentially useful. This said, however, there was still a clear preference for Facebook due to its ubiquity and ease of use. It is interesting to note that, this self-reflection of Yammer usage, for some reason, contradicts with the actual digiMe usage data generated by Google Analytics, which reported that Yammer was amongst the most popularly used apps in digiMe (see Table 2).

There were differences between the Year 4 and Year 5 undergraduate medical student cohorts with Year $4 \mathrm{~s}$ generally being more positive about their experiences with digiMe. Given that the Year 5 students were more established in their Facebook communities, digiMe was regarded by some of them $(n=13)$ as a waste of time, irritating or of no benefit. On the other hand, the Year 4 cohort showed a stronger belief that the digiMe Yammer community had a potential to be useful in their future learning and clinical practice. At the same time, the Year 4 users demonstrated a stronger awareness of the web and mobile apps useful for learning, teaching, clinical or professional practice, and a stronger desire for access to a recommended collection of apps in the future (particularly those for clinical learning and clinical practice).

\section{5. digiMe Usage Recorded through Google Analytics}

The report obtained through Google Analytics provides statistics on the general usage of the digiMe website during the project period. It revealed that there were a total of 129 registered digiMe users, of whom 35 added at least one app as a favourite and 4 users set book marks. Most users accessed digiMe using a desktop computer $(88.43 \%)$. There were a large number of users who have visited the site only once $(n=425)$ or twice $(n=133)$. Given that there are only 129 registered users, these are most likely to be anonymous users who have visited public pages without signing in. Further investigation would need to be conducted to determine the reasons why this many visitors did not sign up. There were, however, significant numbers of users visiting more often. The number of visits per computing device revealed that 120 devices returned 9-14 times, 125 devices returned 15-25 times, 55 devices returned 26-50 times, and 14 devices returned 51-100 times. An average of four pages were viewed each visit per computing device (see Table 3 ).

Since these visitation numbers are considerably greater than the number of total website visits $(N=1115)$, it is likely that Google Analytics has had problems tracking individual users over the full time period. For instance, activities such as clearing browser cookies will hinder this tracking. While this is the case, the numbers can still be used as a rough guide as to how frequently visitors used digiMe. 
Table 2. Top 10 popularly used digiMe apps identified by Google Analytics.

\begin{tabular}{ccccccc}
\hline Page & $\begin{array}{c}\text { Page } \\
\text { Views }\end{array}$ & $\begin{array}{c}\text { Unique Page } \\
\text { Views }\end{array}$ & $\begin{array}{c}\text { Average Time on } \\
\text { Page }\end{array}$ & Entrances & $\begin{array}{c}\text { Bounce Rate } \\
\text { (\%) }\end{array}$ & Exit (\%) \\
\hline Yammer & 165 & 99 & $0: 02: 43$ & 4 & 100 & 24.85 \\
Facebook & 104 & 35 & $0: 01: 47$ & 11 & 9.09 & 16.35 \\
Dropbox & 95 & 42 & $0: 02: 21$ & 4 & 50 & 11.58 \\
400-sbas & 86 & 58 & $0: 01: 53$ & 2 & 0 & 10.47 \\
CloudOn & 62 & 37 & $0: 02: 45$ & 1 & 0 & 12.90 \\
My Medical Tutor & 62 & 50 & $0: 00: 56$ & 0 & 0 & 6.45 \\
OSCE Trainer & 61 & 50 & $0: 01: 18$ & 0 & 0 & 3.28 \\
Weebly & 55 & 42 & $0: 03: 40$ & 11 & 81.82 & 45.45 \\
Audacity & 49 & 41 & $0: 01: 49$ & 3 & 66.67 & 12.24 \\
Clinical presentations & 42 & 31 & $0: 03: 10$ & 0 & 0 & 19.05 \\
\hline
\end{tabular}

Note: Bounce is when a visitor enters the site on this page, and leaves without visiting another page. A bounce rate of $50 \%$ is considered normal for websites.

Table 3. Count of visits to the website.

\begin{tabular}{ccc}
\hline Count of Visits & Visits & Pageviews \\
\hline $9-14$ & 120 & 415 \\
$15-25$ & 125 & 499 \\
$26-50$ & 55 & 228 \\
$51-100$ & 14 & 214
\end{tabular}

Note: 1-8 visits have not been recorded here due to lack of significance. The 'Visits' column represents different computing devices.

Regarding the type of devices used by digiMe users, tablet $(5.38 \%, n=60)$ and mobile $(6.19 \%, n=69)$ visits represent a relatively small proportion of the overall site traffic. This figure is smaller than that was anticipated by the research team at an earlier stage of the research [3]. The majority of the visits were from desktop computers $(88.43 \%, n=986)$. Regarding the operating systems used, the most popular systems were Windows with 701 visits and Macintosh (OS X) with 283 visits. The other operating systems used included iOS with 88 visits, Android with 38 visits, BlackBerry with 3 visits, and Linux with 2 visits. Also, it is worth noting that contrary to normal usage trends, the most popular web browser used was Safari (318 visits), followed by Internet Explorer (296 visits), Chrome (242 visits), Firefox (214 visits), Android Browser (34 visits), IE with Chrome Frame (8 visits), and BlackBerry ( 3 visits). The reason behind this browser choice would need to be further investigated, along with further testing of digiMe operation in Safari running on both Windows and Macintosh (OS X).

The most popularly used apps were Yammer, with 165 page views and 99 unique page views; Facebook with 104 page views and 35 unique page views; and Dropbox with 95 page views and 42 unique page views. A page view indicates when a visitor views a page regardless of whether they have viewed it previously, and a unique page view only counts one view per visitor when a visitor views a page. The usage of the top 10 popularly used apps are shown in Table 2 . The 10 least popular apps, on the other hand, included: Google Plus (six page views), Kindle Reader (six page views), MedCalc (six page views), Research Gate (six page views), Twitter (six page views), Indigenous Health (four page views), Epocrates (three page views), Face time (three page views), YouTube (three page views), and Prezi (one page view).

\section{Discussion}

In this "connected age" [20], technologies that enable connectivity have demonstrated their potential to benefit education at different levels and in different areas. In light of the connectivism theory [6], digiMe was developed with an aim to promote collaborative and peer supported learning. Connectivity to peers was valued by digiMe users. While this reflection is not significant in the participants' self-reported usage of the digiMe community social/professional networking tool, Yammer, it is evident in the Google Analytics reported digiMe app usage, which indicates that Yammer 
was amongst the more popularly used apps. It can be said that this project to some extend helped enhance an awareness of the potential of digiMe, as an example of Web 2.0 supported websites, in building connective and collaborative learning and practice. At the same time, the value of connectivity was evident in the participants' comments which referred to connection to peers via Facebook since early years in their course. This is also demonstrated with $40 \%(n=26)$ of the participants using social networking tools, like Facebook, six or more times per week. This active and spontaneous adoption is also observed in other studies [21,22].

In light of the domestication theory $[7,11]$, digiMe was introduced to and evaluated by the medical students and health professionals in this study. At the pre-intervention phase, potential users' input was sought and used to direct the selection of Web 2.0 tools/apps and the design of digiMe [3]. At the development and post-intervention phase, as being reported in this paper, the participants were offered the opportunity to use the website and its associated Web 2.0 tools/apps for a period of time. The study is shaped by the domestication theory in that, it gave emphasis to the role of users, allowed them the opportunity to use and adapt to the tools, and sought their feedback to support future website development [11]. For instance, with consideration of the participants' feedback in the earlier phase, digiMe was developed to be compatible with different mobile devices and for different web browsers. This capacity was valued by the participants in this later phase of the study. In addition to providing a platform for interactions and information exchange, as what has been provided by existing websites [2,14], digiMe was designed with the focus on both connective and individualised learning. That is, it offers the capacity to allow users to select a range of Web 2.0 tools that they believe to be the most useful in supporting their learning and professional practice.

Whether the context is clinical practice or medical learning and teaching, successful adoption of new technologies is dependent upon a multitude of factors ranging from executive vision and support, willingness and capacity of expected participants, technological constraints, fitness for purpose and context, appropriate integration into workflow, learning or practice, and finally training and support. From a teaching perspective, learning design incorporating apps and Web 2.0 technologies including social media, can be considered where the "technologies support the content, pedagogical, modality ... and synchronicity requirements of the learning tasks." [8] (p. 177). From a learners' perspective, this study recognises the importance of the users' needs, willingness, capacity and preferences [3,23]. A careful consideration and user analysis is crucial for successful implementation of any Web 2.0 technologies. As demonstrated in this study, the design of digiMe reflects the desire of potential users in using mobile devices, as found in the needs analysis at the earlier stage of the research [3]. However, as it was demonstrated through the Google Analytics report, the majority of the medical students and teaching staff used digiMe through desktop computers, despite of their earlier expressed desire to adopt mobile devices. In a way, this Stage 2 of the research further proved the mismatch between users' desires and their actual practice, as what was claimed in the earlier publication [3].

Also, the medical students' year group appeared to be an influential factor for the implementation and adoption of digiMe. Stage 1 of the project saw a need from the potential users in adopting such a tool to support peer learning [3], and digiMe was then built in Stage 2 to respond to this need. The post-intervention survey, however, revealed that students in later year groups have already developed mature communication channels through other means, for instance, Facebook. Therefore, in addition to recognising the needs, the time of implementation for such tools would also need to be taken into consideration [24]. digiMe would have gained more active adoptions if it were introduced to medical students in their earlier study years. Lastly, in the implementation process, support from lecturers and institutions is paramount, and approaches will need rethinking to help learners "navigate this complex and interconnected landscape" [1] (p. 2).

\section{Conclusions}

Connected learning is in its infancy. It is, however, demonstrating an enhanced capacity to support medical education, due to the easier access to Web 2.0 tools which are freely available. This, in turn, can 
facilitate a more individual and learner centric approach where learners create their own learning path. With an awareness of this capacity, this project developed, trialed and evaluated a Web 2.0 supported website: digiMe, with undergraduate medical students and health professionals as the target audience. This paper reported the structure of the website, the functionalities it offers, as well as the apps that are associated with the website. It also provided reports on the findings from the post-intervention survey and website usage data generated by Google Analytics. The results of the study reflects that digiMe is still a website in development, and to obtain more active adoption it would need to be introduced to the potential user group earlier in their study. At the same time, the characteristics of the target users and the environments within which they work are all factors that need to be considered. The participants' evaluation on digiMe, along with the statistical data collected on the website usage, provide useful insights for further development of digiMe or other Web 2.0 supported websites with an aim to support connected learning.

Acknowledgments: The project was funded by the Health Workforce Australia (HWA). Ethics approval was obtained from the Tasmanian Social Sciences Human Research Ethics Committee (Reference Number H0012730).

Author Contributions: All authors contributed to the conduct and design of the research. Si Fan drafted the manuscript, Jan Radford and Debbie Fabian wrote part of the manuscript and contributed to the revision and editing of the manuscript.

Conflicts of Interest: The authors declare no conflicts of interest.

\section{References}

1. Educause Learning Initiative. Connected Learning 7 Things You Should Know about .... Available online: https://library.educause.edu/resources/2013/5/7-things-you-should-know-about-connectedlearning (accessed on 17 August 2017).

2. ThinkGP. News and Education for Health Professionals. Available online: http://thinkgp.com.au/ (accessed on 17 August 2017).

3. Fan, S.; Radford, J.; Fabian, D. A Mixed-Method Research to Investigate the Adoption of Mobile Devices and Web 2.0 Technologies among Medical Students and Educators. BMC Med. Inform. Decis. Mak. 2016, 16, 43. [CrossRef] [PubMed]

4. Cheston, C.C.; Flickinger, T.; Chisolm, M.S. Social Media Use In Medical Education: A Systematic Review. Acad. Med. 2013, 88, 893-901. [CrossRef] [PubMed]

5. Le, Q.; Fan, S. Computer-Supported Learning in the Context of Health Education. In Proceedings of the Third International Conference on Innovations in Learning for the Future 2010: E-Learning, Istanbul, Turkey, 2010; pp. 330-339.

6. Siemens, G. Connectivism: A Learning Theory for the Digital Age. Available online: http://er.dut.ac.za/ handle/123456789/69 (accessed on 17 August 2017).

7. Silverstone, R.; Haddon, L. Design and the Domestication of Information and Communication Technologies: Technical Change and Everyday Life. In Communication by Design: The Politics of Information and Communication Technologies; Mansell, R., Silverstone, R., Eds.; Oxford University Press: Oxford, UK, 1996; pp. 44-74. ISBN 978-0-19828-9418.

8. Bower, M.; Hedberga, J.G.; Kuswaraa, A. A Framework for Web 2.0 Learning Design. Educ. Media Int. 2010, 47, 177-198. [CrossRef]

9. van Harmelen, M. Personal Learning Environments Make a Step Forward. Available online: http:/ /fm. schmoller.net/2006/07/personal_learni.html (accessed on 17 August 2017).

10. Stephenson, K. What Knowledge Tears Apart, Networks Make Whole. Available online: http://www. netform.com/html/icf.pdf (accessed on 17 August 2017).

11. Hynes, D.; Richardson, H. What Use is Domestication Theory to Information Systems Research? In Handbook of Research on Contemporary Theoretical Models in Information Systems; Dwivedi, Y.K., Lal, B., Williams, M.D., Schneberger, Y.K., Wade, M., Eds.; IGI Global: Hershey, PA, USA, 2009; pp. 482-494. ISBN 987-1-60566-659-4.

12. Fan, S.; Radford, J. GPaedia: A Web 2.0 Technology Enhanced Digital Habitat to Support The General Practice Learning Community. Focus Health Prof. Educ. 2015, 16, 3-15. [CrossRef] 
13. Fan, S.; Radford, J.; Brown, J. A Virtual Learning Community Supporting the General Practice Education and Training in Australia. In Proceedings of the 5th International Conference on Education and New Learning Technologies, Barcelona, Spain, 2013; pp. 626-633.

14. e-healthspace. e-healthspact: Connect with Other Doctors. Available online: http://www.e-healthspace. com.au/home.php (accessed on 17 August 2017).

15. auspharmacist. Auspharmacist. Available online: http://www.auspharmacist.net.au/ (accessed on 17 August 2017).

16. Likert, R.A. Technique for the Measurement of Attitudes. Arch. Psychol. 1932, 22, 55.

17. Pallant, J. SPSS Survival Manual; McGraw-Hill Education: Berkshire, UK, 2013; ISBN 978-0-33526-2588.

18. Charmaz, K. Constructing Grounded Theory, 2nd ed.; Sage: London, UK, 2014; ISBN 978-0-85702-9133.

19. Google Analytics. Get Start with Google Analytics. Available online: https://analytics.google.com/ (accessed on 17 August 2017).

20. Watts, D.J. Six Degrees: The Science of a Connected Age. W.W. Norton \& Co.: New York, NY, USA, 2004.

21. Cartledge, P.; Miller, M.; Phillips, B. The Use of Social-networking Sites in Medical Education. Med. Teach. 2013, 35, 847-857. [CrossRef] [PubMed]

22. Gray, K.; Annabell, L.; Kennedy, G. Medical Students' Use of Facebook to Support Learning: Insights from Four Case Studies. Med. Teach. 2010, 32, 971-976. [CrossRef] [PubMed]

23. Venkatesh, V.; Croteau, A.M.; Rabah, J. Perceptions of Effectiveness of Instructional Uses of Technology in Higher Education in an Era of Web 2.0. In Proceedings of the 47th Hawaii International Conference on System Science, Waikoloa , HI, USA, 2014; pp. 110-119.

24. Fan, S. Significance of the Web as a Learning Resource in an Australian University Context. Ph.D. Thesis, University of Tasmania, Tasmania, August 2011.

(C) 2017 by the authors. Licensee MDPI, Basel, Switzerland. This article is an open access article distributed under the terms and conditions of the Creative Commons Attribution (CC BY) license (http:/ / creativecommons.org/licenses/by/4.0/). 